\title{
All-scale Architecturing of Microstructure in Chalcogenide Thermoelectric Materials
}

\author{
Songting Cai ${ }^{1}$, Yubo Luo $^{2,3}$, Xiaobing $\mathrm{Hu}^{1,4}$, Mercouri G. Kanatzidis ${ }^{2,5}$ and Vinayak P. Dravid ${ }^{1,4}$ \\ 1. Department of Materials Science and Engineering, Northwestern University, Evanston, IL, USA. \\ 2. Department of Chemistry, Northwestern University, Evanston, IL, USA. \\ 3. School of Materials Science and Engineering, Nanyang Technological University, Singapore. \\ 4. NUANCE Center, Northwestern University, Evanston, IL, USA. \\ 5. Materials Science Division, Argonne National Laboratory, Argonne, IL, USA.
}

Thermoelectric materials can realize direct conversion between heat and electricity, therefore is regarded as a crucial candidate for clean power generation and refrigeration [1]-[2]. The energy conversion efficiency of such materials is determined by dimensionless figure of merit, $Z T=S^{2} \sigma T /\left(\kappa_{e}+\kappa_{L}\right)$, where $S$ is the Seebeck coefficient, $\sigma$ is the electrical conductivity, and $T$ is the absolute temperature. Many studies have recently highlighted the role of microstructural constituents, such as defects, on decreasing the lattice thermal conductivity via phonon scattering with different frequencies [2].

Herein, we present advanced electron microscopy of all-scale architecture construction of two wellknown chalcogenide thermoelectric materials: PbSe and SnSe. Figure 1. (a)-(c) illustrate S/TEM analyses of Cd-alloyed PbSe (PbSe-10\%CdSe) sample. Decent amount of faceted CdSe precipitates can be observed within the PbSe matrix. With the help of probe aberration-corrected STEM, "super-structured" interface between the matrix and interface is observed. Such architecture gives rise to significant lattice strain, therefore may contribute to the decrease in the lattice thermal conductivity of the materials. As shown in Figure 2. (a), dispersed $\mathrm{Ag}_{8} \mathrm{SnSe}_{6}$ precipitates ranging from $\sim 20$ to $60 \mathrm{~nm}$ has been introduced in polycrystalline SnSe matrix. The chemical analysis with EDS reveals that Sn, Se and Ag are distributed uniformly throughout the matrix, while the precipitates are rich in Ag but deficient in Sn and Se. Typical HRTEM imaging along with elected area diffraction (SAED) analyses are all consistent with the crystal structure of matrix (SnSe, space group: Pnma) and the precipitates $\left(\mathrm{Ag}_{8} \mathrm{SnSe}_{6}\right.$, space group: Pmn2 $\left.{ }_{1}\right)$, with a low-index orientation relationship. The introduction of nano-size precipitate significantly depresses the lattice thermal conductivity. As a result, an enhanced $Z T$ of $\sim 1.3$ is obtained [3].

To summarize, multiple-scale microstructure plays a significant role in phonon scattering and charge transport modification. Advanced electron microscopy can reveal both qualitative \& quantitative elemental information as well as crystal structure relationship of the microstructure, therefore provide vital insights on heat treatment design and kinetics study of thermoelectric materials [4].

\section{References:}

[1] Zhang, X. \& Zhao, L.-D. J. Materiomics 1 (2015), 92.

[2] Tan, G., Zhao, L.-D. \& Kanatzidis, M. G. Chem. Rev. 116 (2016), p. 12123.

[3] Luo, Y. et al. Adv. Energy Mater. 0, 1803072

[4] This work was supported by the Department of Energy, Office of Science, Basic Energy Sciences under Grant DE-SC0014520, made use of the EPIC facility of Northwestern University's NUANCE Center, which has received support from the Soft and Hybrid Nanotechnology Experimental (SHyNE) Resource (NSF ECCS-1542205); the MRSEC program (NSF DMR-1121262) at the Materials 
Research Center; the International Institute for Nanotechnology (IIN); the Keck Foundation; and the State of Illinois, through the IIN.

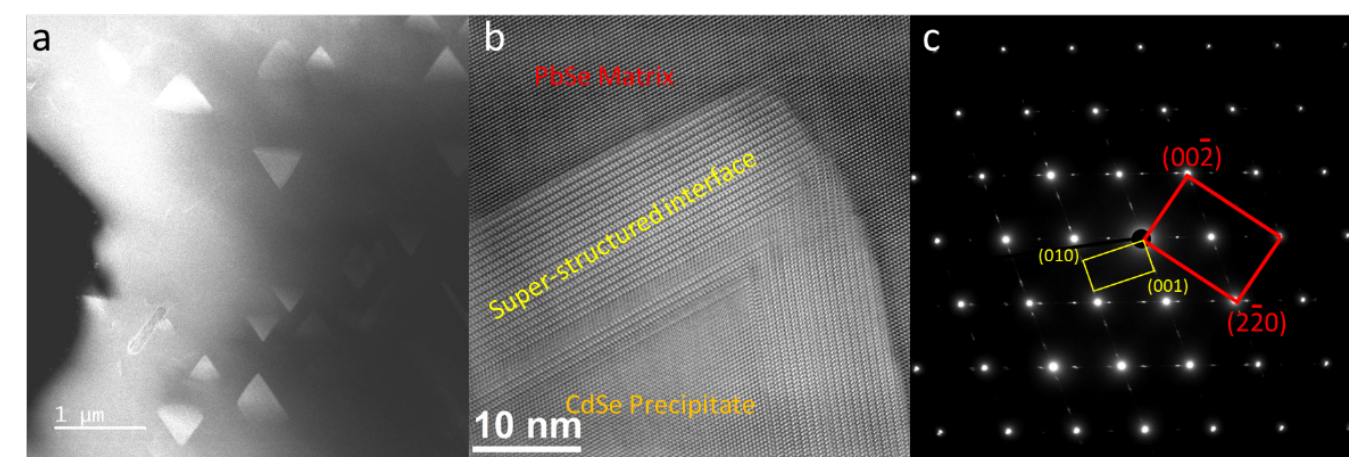

Figure 1. S/TEM analyses of PbSe-10\%CdSe. (a) HAADF image. (b) Atomic-resolution bright field (BF) STEM image (c) Selected area diffraction pattern of (b).
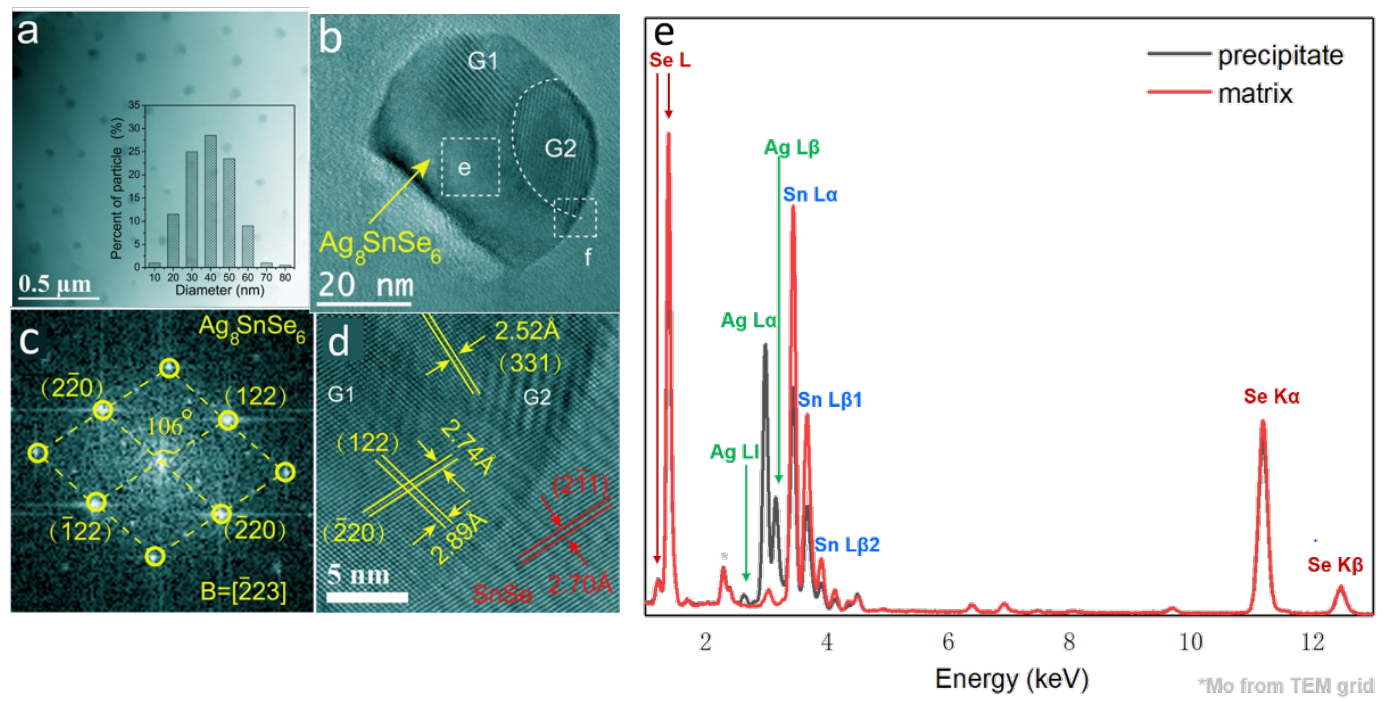

Figure 2. (a) STEM annular bright field (ABF) image of $\mathrm{SnSe}-\mathrm{Ag}_{8} \mathrm{SnSe}_{6}(\mathrm{SnSe}-\mathrm{STSe})$. (b) High resolution TEM image of STSe precipitate. Two parts with different orientation are labeled with G1 and G2. (c) Fast Fourier transform (FFT) pattern of the box region (e). (d) HREM image of region G2. (e) EDS spectrum comparison between the matrix and the precipitate [3]. 